\title{
Evolução das políticas públicas frente à redução da mortalidade infantil e na infância
}

\section{no Brasil}

\author{
The evolution of public policies toward the reduction of child and under-five child mortality in
}

Brazil

Evolución de las políticas públicas frente a la reducción de la mortalidad infantil y en la infancia en

Brasil

Recebido: 19/08/2021 | Revisado: 01/09/2021 | Aceito: 04/09/2021 | Publicado: 12/09/2021

Cristiane da Silva Ramos Marinho
ORCID: https ://orcid.org/0000-0003-3825-3057
Universidade Federal do Rio Grande do Norte, Brasil
E-mail: cristiane_ramos@hotmail.com
Maria Ângela Fernandes Ferreira
ORCID: https://orcid.org/0000-0002-6142-948X
Universidade Federal do Rio Grande do Norte, Brasil
E-mail: mangelaf50@gmail.com

\section{Resumo}

O presente artigo consiste em uma revisão narrativa da literatura sobre as políticas públicas de saúde e de âmbito social à criança no Brasil que contribuíram para a redução da Taxa de Mortalidade Infantil e na Infância. A busca foi feita nas bases de dados Medline, Lilacs, PAHO, em documentos governamentais e no Google Acadêmico, envolvendo o período de 1973 a 2015 . A coleta dos dados ocorreu entre novembro de 2018 e junho de 2019 a partir dos temas mortalidade infantil e na infância no Brasil, tendo sido incluído na revisão todos os artigos completos e documentos governamentais e leis que abordaram de forma direta ou indireta a mortalidade infantil e/ou na infância publicados no idioma Português. As diversas estratégias, programas e políticas públicas de saúde e de âmbito social desenvolvidas ao longo dos anos foram essenciais para o alcance das reduções nas taxas de mortalidade infantil e na infância no Brasil. Os avanços obtidos são motivos de comemoração, mas o cenário atual continua sendo um desafio quanto à manutenção da redução dessas taxas.

Palavras-chave: Mortalidade; Saúde da criança; Política pública.

\begin{abstract}
This article consists of a narrative review of the literature on public health and social policies for children in Brazil that contributed to the reduction of the Child and Under-five child Mortality Rate. Search was carried out in Medline, Lilacs, PAHO databases, in government documents and on Google Scholar, covering the period from 1973 to 2015. Data collection occurred between November 2018 and June 2019 from the themes child and under-five child mortality in Brazil, including in the review all full-text articles, government documents and laws that directly or indirectly addressed child and/or under-five child mortality published in the Portuguese language. The numerous strategies, programs and public health and social policies developed over the years were essential for achieving reductions in child and under-five child mortality rates in Brazil. The advances made are cause for celebration, however the current scenario remains a challenge in terms of maintaining the reduction of these rates.
\end{abstract}

Keywords: Mortality; Child health; Public policy.

\section{Resumen}

El presente artículo consiste en una revisión narrativa de la literatura acerca de las políticas públicas de salud y del ámbito social a los niños en Brasil que han contribuido para la reducción de la Tasa de Mortalidad Infantil y en la Infancia. La búsqueda se hizo en las bases de datos Medline, Lilacs, PAHO, en documentos gubernamentales y en Google Académico, considerando el período de 1973 a 2015. La recolecta de datos sucedió entre noviembre de 2018 y junio de 2019, a partir de los temas mortalidad infantil y en la infancia en Brasil, y fueron incluidos en la revisión todos los artículos completos y documentos gubernamentales y leyes que, de forma directa o indirecta, tratan de la mortalidad infantil y/o en la infancia, publicados en el idioma Portugués. Las diversas estrategias, programas y políticas públicas de salud en ámbito social desarrollados a lo largo de los años fueron esenciales para el alcance de las reducciones en las tasas de mortalidad infantil y en la infancia en Brasil. Los avances obtenidos son motivos de celebración, pero el escenario actual sigue siendo un desafío cuanto a la manutención de la reducción de esas tasas.

Palabras clave: Mortalidad; Salud del niño; Política pública de salud. 


\section{Introdução}

A história da saúde da criança no Brasil vem sendo construída há décadas, tendo sofrido várias transformações. Até o início dos anos 1980, a área encontrava-se agregada à da saúde da mulher. Somente em 1984, a partir da instituição do Programa de Assistência Integral à Saúde da Criança (PAISC) que se começa a observar o desenvolvimento de ações mais específicas voltadas exclusivamente para a criança (Brasil, 2011a). Naquele momento, o país apresentava elevadas taxas de mortalidade infantil (TMI), taxas de mortalidade de crianças menores de 1 ano, e na infância (TMInf), taxas de mortalidade de crianças menores de 5 anos (Ipea, 2014), reflexo de um cenário de falta de prioridade à atenção à saúde da criança.

Acordos internacionais e o desenvolvimento de uma série de ações, tanto na área da saúde quanto na área social, resultaram no alcance de melhores condições de vida para a população e consequentemente em reduções consideradas nas TMI e TMInf no país que puderam ser constatadas no início do século XXI. No entanto, apesar de todo o avanço, as TMI e TMInf no Brasil ainda se encontram com níveis elevados, bem superiores aos de países desenvolvidos, e com distribuição heterogênea entre as regiões brasileiras, o que continua a requerer maior ênfase em políticas, programas e ações que contribuam para a redução desses indicadores (Ipea, 2014).

O desenvolvimento e a monitoragem de estratégias políticas eficazes na melhoria dos determinantes sociais que promovem as desigualdades são mecanismos apontados como caminhos para a redução da mortalidade infantil (Cacau et al., 2015). Nas últimas décadas, o Brasil vinha conseguindo uma redução das desigualdades regionais e das disparidades sociais, embora esta última em menor grau (Caldas et al., 2017), mas desde 2015, em meio a uma forte crise política e econômica, vem se observando um aumento da pobreza e como consequência, em 2016, já pôde ser observado um aumento na TMI e na TMInf no país, de acordo com dados do próprio ministério da saúde (Brasil, 2020a).

Medidas de austeridade fiscal, que atuam na redução dos gastos com proteção social, foram implantadas, com destaque para a Emenda Constitucional 95 (EC95/2016), impactando diretamente nesses indicadores. Vale ressaltar que a pobreza é um dos determinantes sociais mais importantes da saúde das crianças, de forma que programas que atuam na sua redução contribuem para a diminuição da morbimortalidade de crianças abaixo dos 5 anos em vários países (Rasella et al., 2018).

Assim, o estímulo dado ao desenvolvimento de políticas públicas que favoreceram a melhora da renda, o aumento de cobertura dos serviços de saúde e da educação contribuíram positivamente para a obtenção de melhores indicadores da saúde infantil no Brasil como um todo (Caldas et al., 2017). Portanto, reduzir a inversa e significante associação entre nível socioeconômico e a mortalidade infantil e na infância é essencial, sendo considerado um desafio que se revela presente tanto entre regiões, como no âmbito dos estados e municípios (Santos et al., 2014).

Diante da importância dessa temática e da perspectiva de desmonte das políticas públicas de saúde e de proteção social, o estudo objetivou realizar um levantamento bibliográfico de artigos e documentos relevantes publicados em bases e bancos de dados eletrônicos por meio de uma revisão narrativa da literatura sobre a evolução dos programas, políticas públicas, estratégias, ações, iniciativas e planos na área da saúde e social que influenciaram na redução das TMI e nas TMInf no Brasil.

\section{Metodologia}

Trata-se de uma revisão narrativa da literatura realizada nas bases de dados MEDLINE (Medcal Literature Analysis and Retrieval System Online), LILACS (Literatura Latino-americana em Ciências de Saúde), PAHO (Pan American Health Organization), em documentos governamentais e no Google Acadêmico acerca das políticas públicas 
de saúde e sociais que contribuíram para a diminuição dos indicadores infantis e na infância no Brasil. Para Cordeiro, Oliveira, Rentería e Guimarães (2007), a revisão da literatura narrativa ou tradicional é um tipo de publicação ampla que apresenta uma temática aberta sobre um determinando assunto. Não apresenta rigor quanto a utilização de protocolo rígido para sua confecção. Realiza busca de fontes de dados de modo não pré-determinado, utilizando -se de seleção arbitrária de artigos.

A busca da literatura foi realizada no período de novembro de 2018 a junho de 2019, a partir dos temas mortalidade infantil e na infância no Brasil, envolvendo o período de 1973 a 2015, tendo sido incluído na revisão todos os artigos completos e documentos governamentais, bem como leis que abordaram de forma direta ou indireta a mortalidade infantil e/ou na infância publicados no idioma Português. Ressalta-se a importância das revisões narrativas por possibilitarem a reunião em um único trabalho de informações gerais/pontuais sobre uma determinada temática abordada.

Os dados foram analisados e interpretados com base na apreciação crítica das informações obtidas, buscando correlacioná-las aos objetivos do estudo.

\subsection{Evolução das políticas públicas à criança no Brasil}

Por muitos anos, a atenção à saúde da criança não foi definida como prioridade no contexto das políticas públicas no Brasil. O processo histórico envolvendo as ações de saúde à criança foi gradual, com suas primeiras intervenções pontuais decorrentes de situações agudas, sendo de caráter curativo e individualizado, com foco direcionado à diminuição do absenteísmo materno no trabalho (Araújo et al., 2014).

Em função da precariedade das ações de saúde e da grave crise econômica, social e política do país, a situação epidemiológica da população brasileira entre as décadas de 1960 e 1970 caracterizou-se por vários problemas sanitários, além do surgimento de uma série de epidemias. Diante disso, vários projetos, programas, planos, redes de atenção, legislações e ações de saúde foram implementados ao longo dos anos. Para ilustrar e nortear a descrição dessas ações foi construída uma linha do tempo com as principais políticas de atenção à saúde da criança a partir do ano de 1973 (Figura $1)$. 
Figura 1 - Evolução das Políticas, Programas, Estratégias, Iniciativas, Planos que influenciaram à saúde da criança no Brasil, 1973 a 2015.

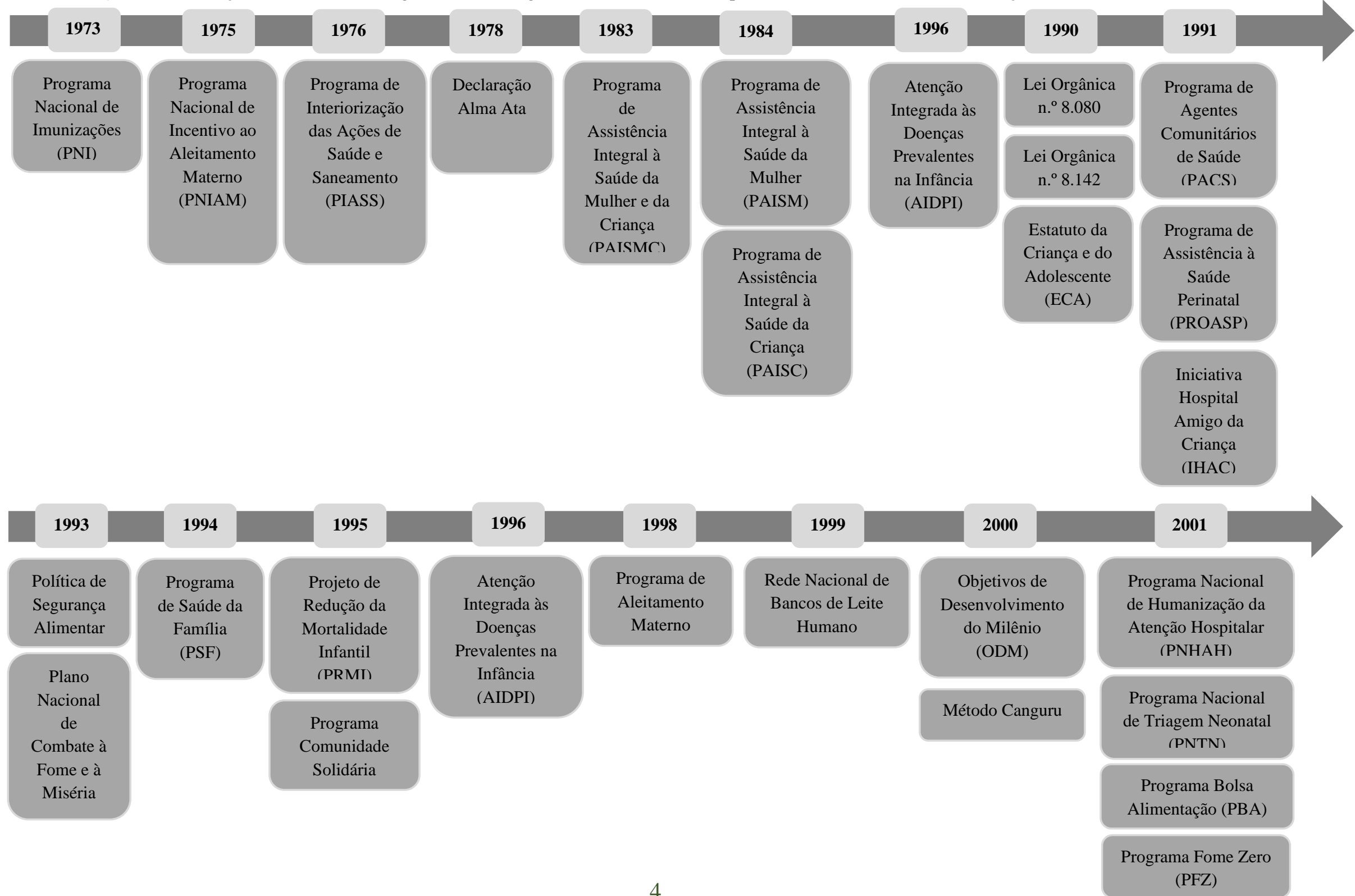


Research, Society and Development, v. 10, n. 11, e474101119584, 2021

(CC BY 4.0) | ISSN 2525-3409 | DOI: http://dx.doi.org/10.33448/rsd-v10i11.19584

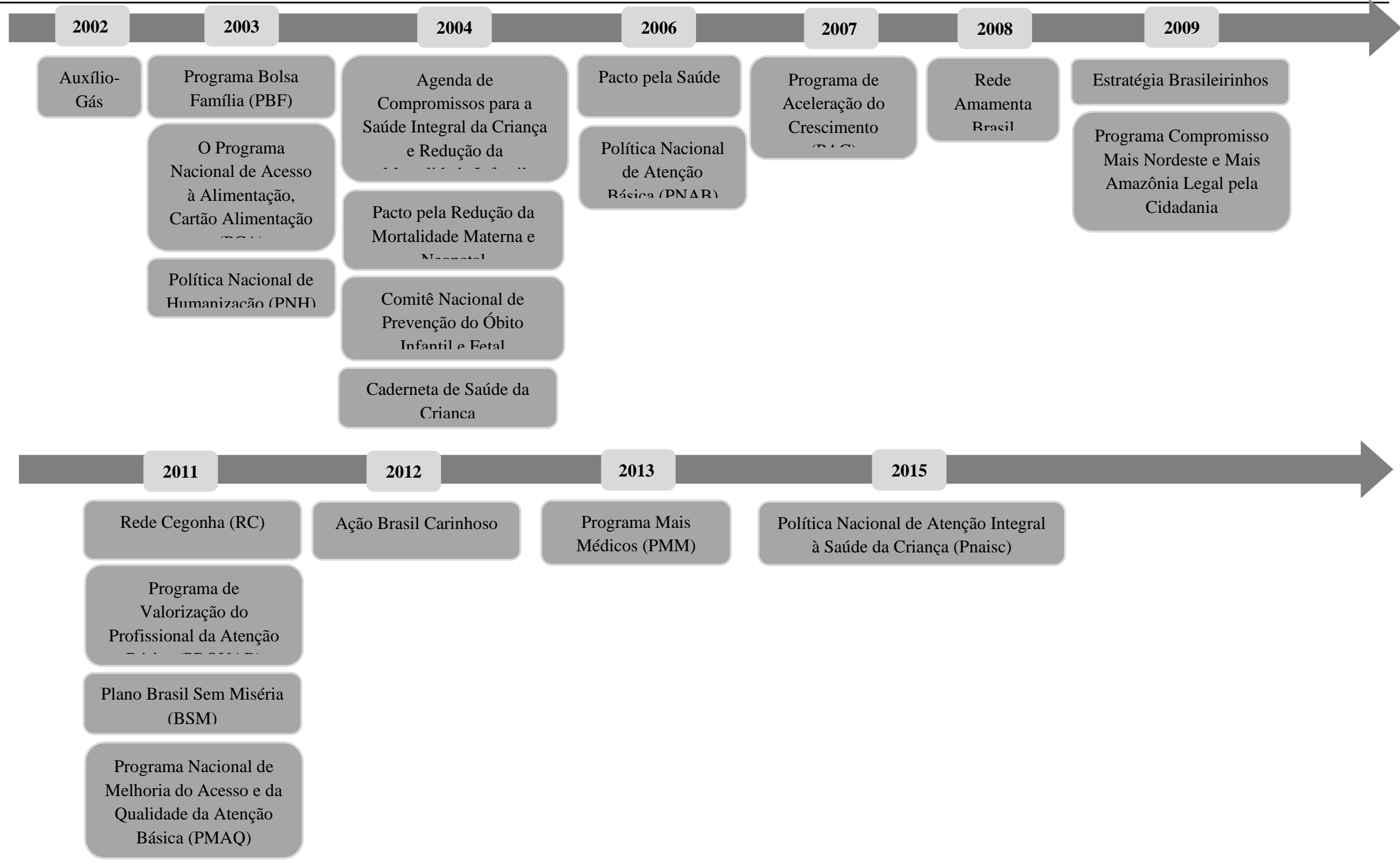

Fonte: Autores. 
Em 1973, diante de uma conjuntura de grave crise sanitária, com altas taxas de mortalidade de crianças por doenças imunopreveníveis, é implantado o Programa Nacional de Imunizações (PNI), como um instrumento de organização e implementação do calendário vacinal no Brasil, objetivando o controle de doenças de grande impacto nas condições de vida da população, atuando de forma primária na prevenção e erradicação de diferentes patologias 9, contribuindo para a melhoria da saúde das crianças, parcela vulnerável ao adoecimento.

Por meio do PNI foi instituída a multivacinação, e em 1980 tem início uma mobilização coletiva entre União, Estados e Municípios para a realização da primeira campanha de vacinação em massa, em um só dia, em todo o território nacional, abrangendo crianças de 0 a 5 anos de idade, independente de vacinação prévia (Nascimento, 2011). Os êxitos alcançados pelo PNI fizeram dele um programa com grande reconhecimento e respeitabilidade por parte da sociedade brasileira e mundial, tornando-o um programa de Saúde Pública de referência para vários países. Um fator indispensável para o sucesso das ações do Programa foi o apoio da população, que oportunizou o alcance de coberturas vacinais adequadas, tanto nas ações de rotina quanto nas campanhas de vacinação (Silva Júnior, 2012).

Com isso, durante muitos anos existiram fortes indícios e indicadores que apontavam para a conquista de "cidadania biomédica" inclusiva por parte da população, em função da oferta pública de considerado número de imunizantes e boa cobertura vacinal nas campanhas, mesmo diante da desigualdade social (Hochman, 2011).

Outros programas de grande relevância para a área da criança foram o Programa Nacional de Saúde MaternoInfantil, instituído em 1975 (Brasil, 2011a) o Programa Nacional de Incentivo ao Aleitamento Materno (PNIAM), em 1981 (Brasil, 2018) e o Programa de Assistência Integral à Saúde da Mulher e da Criança (PAISMC), em 1983, desmembrado no ano seguinte em Programa de Assistência Integral à Saúde da Mulher (PAISM) e Programa de Assistência Integral à Saúde da Criança (PAISC) (Brasil, 2011a). A partir da instituição do PAISC a saúde da criança deixa de ser diretamente ligada à saúde materna e passa a receber atenção mais específica, com foco prioritário nas crianças.

Em relação à área social, em agosto de 1976 foi criado o Programa de Interiorização das Ações de Saúde e Saneamento (PIASS) com a finalidade de expandir a cobertura de ações de saúde/saneamento a populações desassistidas, propondo a implantação de uma estrutura básica de saúde pública para municípios com menos de 20.000 habitantes. Inicialmente o foco seria a região Nordeste, e posteriormente haveria expansão para todo o território nacional. No entanto, desde o início, a atuação do PIASS não se limitou a Região Nordeste, estendendo-se pelo 'Polígono das Secas', incluindo o norte de Minas e o Maranhão. Apesar dos investimentos não terem sido distribuídos de forma equitativa entre os estados envolvidos, havendo maior priorização dos gastos entre os estados do Rio Grande do Norte, Pernambuco, Alagoas, Bahia e Minas Gerais, mesmo assim, o PIASS conseguiu promover ampliação da rede de saúde. Ao final de 1978, estavam implantados e funcionando 642 centros de saúde e 1.250 postos de saúde, com um potencial de cobertura de 6.400 .000 pessoas em 699 municípios, o que correspondia a 56\% do total da sua área de abrangência (Escorel, 1999).

Levando em consideração que até aquele momento a assistência oferecida a população se limitava ao Instituto Nacional de Assistência Médica da Previdência Social (INAMPS), com escassos hospitais públicos, alguns poucos centros de saúde nas capitais e nas cidades polo, além de precários minipostos de saúde nos municípios do interior dos estados, a nova estrutura de atenção básica possibilitou uma melhora quanto a oferta dos serviços de saúde, além de contribuir com as campanhas de multivacinação concebidas para controlar as doenças imunopreveníveis, que eram responsáveis pela morbimortalidade de parcela considerável das crianças.

Nesse contexto, o movimento sanitário brasileiro, que atuou na luta em defesa da universalização da saúde pública e dos direitos da criança, ganha força exigindo o enfrentamento de distorções estruturais do sistema de saúde, 
buscando a superação do grave quadro de desigualdades em saúde no país. Assim, conquista-se a promulgação da Constituição Federal em 1988 e, na sequência, a criação do Sistema Único de Saúde (SUS) e a homologação das Leis Orgânicas n. ${ }^{\circ} 8.080$ e n. ${ }^{\circ} 8.142$ de 1990 (Magalhães et al., [2018?]). O SUS foi uma grande conquista da sociedade brasileira, criado para promover a justiça social e superar as desigualdades na assistência à saúde da população, universalizando o atendimento e melhorando os indicadores de saúde das crianças (Brasil, 2011b). Ancorado nos princípios de universalização, equidade e integralidade, junto à participação social, o sistema de saúde brasileiro que atendia pequena parcela da população passou a assistir à todos os brasileiros (Brasil, 2018).

A chegada dos anos 1990 trouxe consigo grandes transformações para o setor saúde no país, na ocasião o país ainda apresentava elevados índices de mortalidade infantil e na infância (Gráficos 1 e 2). No começo da nova década, o Brasil e outros 158 estados-membros da Organização das Nações Unidas (ONU), durante a Reunião da Cúpula Mundial em Favor da Infância, constataram que as metas traçadas na Declaração de Alma Ata, instituída em 1978, que versava sobre o lema "Saúde para todos no ano 2000", não haviam sido atingidas, o que os levou os países membros a assinarem a Declaração Mundial sobre a Sobrevivência, Proteção e Desenvolvimento da Criança (Brasil, 1997). Nesse contexto é criado o Estatuto da Criança e do Adolescente (ECA), passando a assegurar às crianças e aos adolescentes uma série de direitos (Brasil, 2005).

Gráfico 1 - Taxas de Mortalidade Infantil no Brasil, utilizando metodologia do Busca Ativa, 1990 a 2018.

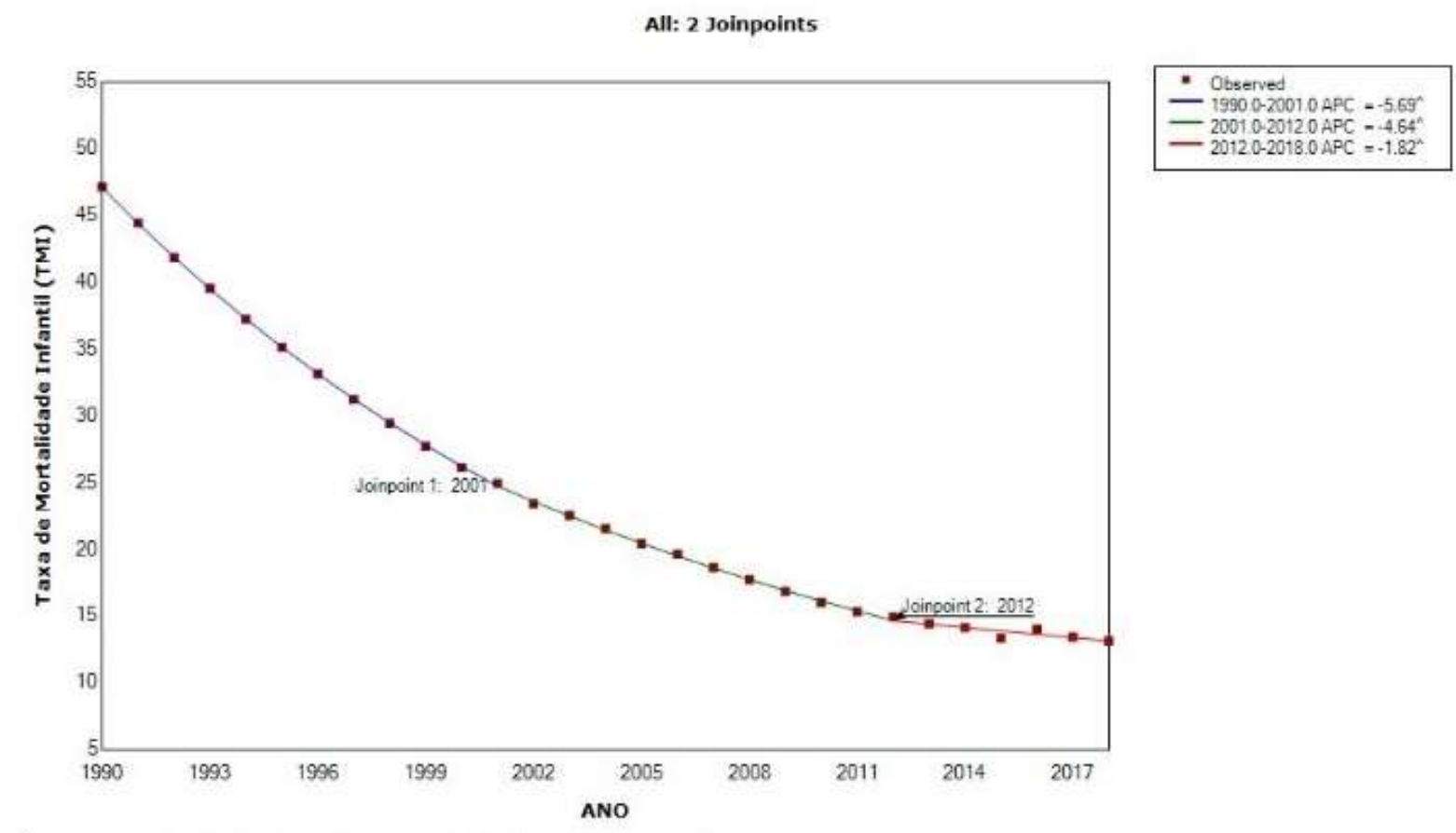

Andicates that the Annual Percent Change (APC) is significantly different from zero at the alphe -0.05 lovel

MS: Ministério da Saúde; CGIAE: coordenação Geral de Informações e Análises Epidemiológicas; SVS: Secretaria de Vigilância em Saúde; SIM: Sistema de Informação sobre Mortalidade; Sinasc: Sistema de Informação sobre Natalidade

Fonte: MS/SVS/CGIAE - SIM/Sinasc e Busca Ativa. 
Gráfico 2 - Taxas de Mortalidade na Infância no Brasil, utilizando metodologia do Busca Ativa, 1990 a 2018.

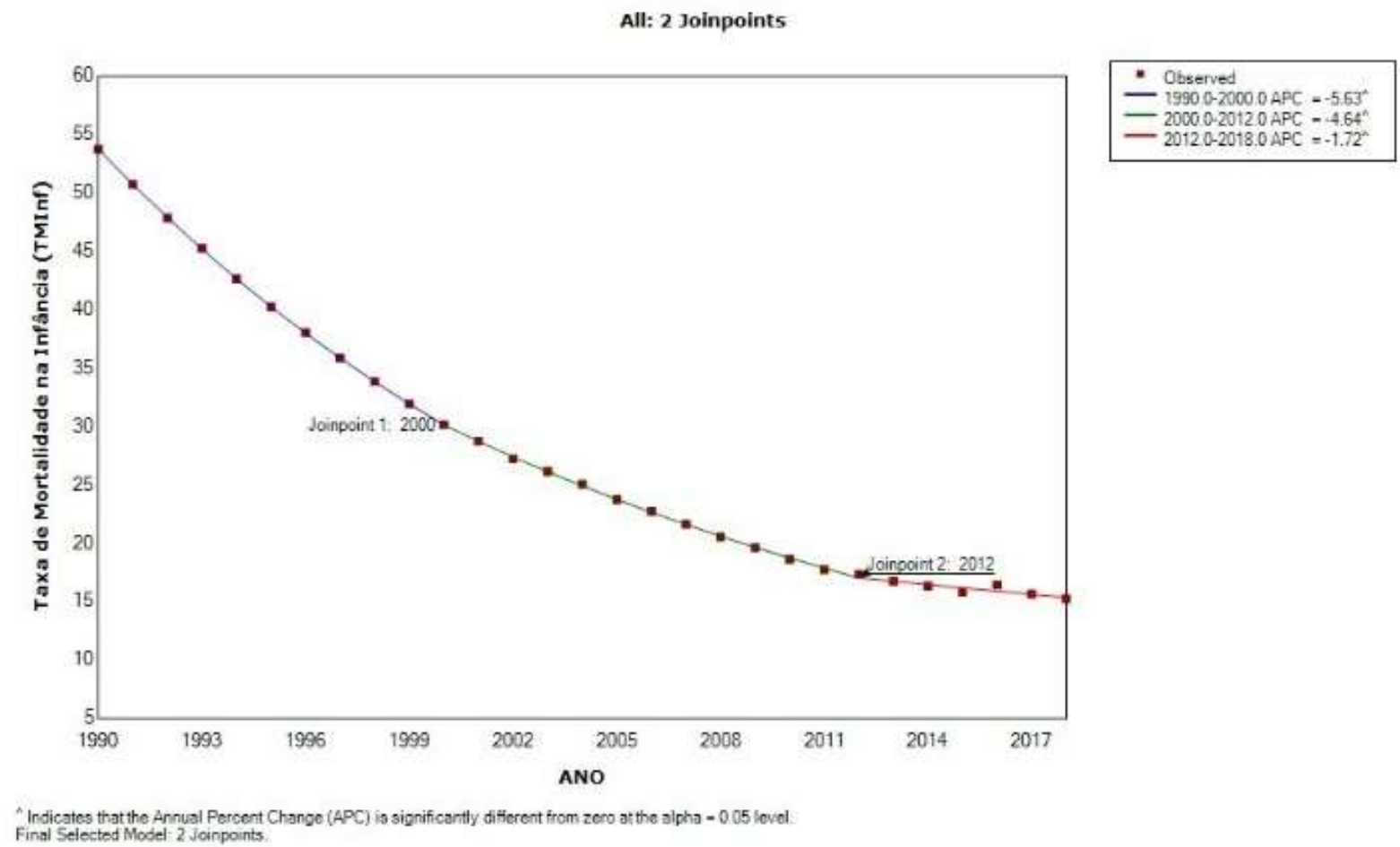

MS: Ministério da Saúde; CGIAE: coordenação Geral de Informações e Análises Epidemiológicas; SVS: Secretaria de Vigilância em Saúde; SIM: Sistema de Informação sobre Mortalidade; Sinasc: Sistema de Informação sobre Natalidade

Fonte: MS/SVS/CGIAE - SIM/Sinasc e Busca Ativa.

Na sequência, em 1991, é criado o Programa de Agentes Comunitários de Saúde (PACS) para melhorar as condições de saúde das comunidades (Brasil, 2020b) e, em seguida, o Programa de Assistência à Saúde Perinatal (PROASP), que buscou organizar a assistência perinatal, instituindo o alojamento conjunto (Costa et al., 2010). O PACS teve um papel decisivo na redução da mortalidade infantil e na infância por meio da ampliação das visitas domiciliares para áreas mais pobres e desvalidas (Viana, Poz, 2005; Magalhães et al., [2018?]).

No decorrer da década de 1990 surgiram algumas políticas de estímulo à prática do aleitamento materno: a Iniciativa Hospital Amigo da Criança (IHAC), em 1991, o Programa de Aleitamento Materno, em 1998, e a Rede Nacional de Bancos de Leite Humano em 1999 (Brasil, 2017a). A IHAC surge como proposta de integrar a assistência, dando autonomia para a mãe e bebê, principalmente, no que se diz respeito ao parto humanizado, aleitamento materno e consequentemente favorecendo à redução de doenças e da TMI (Araújo et al., 2014). O incentivo ao aleitamento materno passou a ser prioridade na gestão nacional do SUS, uma vez que várias evidências científicas começaram a surgir comprovando a superioridade da amamentação quando comparada a outras formas de alimentação, particularmente em crianças menores de 1 ano.

A partir de 1993, observa-se o desencadeamento da formação de milhares de comitês de solidariedade e combate à fome, fortalecendo o Conselho Nacional de Segurança Alimentar (Consea) e oportunizando a instituição da Política de Segurança Alimentar e do Plano Nacional de Combate à Fome e à Miséria (Silva, Grossi, França, 2010; Guimarães, Silva, 2020). Não se pode negar a importância de tais iniciativas, no entanto, ainda tratavam-se de ações muito pontuais e fragmentadas, uma vez que o combate à fome e a miséria envolvem uma série de determinantes sociais, requerendo 
políticas públicas diretamente associadas a construção de uma rede de proteção social. Assim, continuou sendo um desafio para o país o alcance desse objetivo, tão importante na redução da mortalidade infantil e na infância.

No ano seguinte, em 1994, foi implantado o Programa de Saúde da Família (PSF) a fim de garantir acesso aos cuidados primários de saúde, com base nos princípios do SUS (Santos, Jacinto, 2017). A implantação do PSF foi um avanço para a saúde pública brasileira por promover a interiorização de categorias profissionais, desenvolver ações de prevenção, promoção e recuperação da saúde de forma ativa e reduzir as desigualdades de saúde no Brasil. Estudo realizado pelo Ministério da Saúde em colaboração com a Universidade de Nova York revela que entre 1990 e 2002, para cada 10\% de aumento na população coberta pelo PSF houve redução média de 4,6\% na TMI (Brasil, 2008a). Dessa feita, o PSF trata-se de uma importante iniciativa para salvar vidas e melhorar a qualidade da atenção à saúde da criança, pela ampliação da cobertura e do acesso aos serviços de saúde, especialmente para as famílias que mais necessitam (Magalhães et al., [2018?]).

Na sequência, em 1995, é lançado o Projeto de Redução da Mortalidade Infantil (PRMI) visando intensificar esforços em favor da infância, por intermédio da união de vários programas e instituições, que promoveram ações de imunização, de combate às doenças infecciosas, desnutrição, promoção ao aleitamento materno, pré-natal e de saneamento básico (Brasil, 2018). Em 1996 foi implantada a estratégia de Atenção Integrada às Doenças Prevalentes na Infância (AIDPI) para a diminuição da morbimortalidade de crianças entre 2 meses a 5 anos de idade (Brasil, 2017b) a partir de uma adequada relação custo/benefício na diminuição da carga de doenças e para melhora das condições de saúde da criança (Magalhães et al., [2018?]).

Na virada do milênio, a ONU e outros 191 países signatários propuseram os Objetivos de Desenvolvimento do Milênio (ODM), como um guia para redução da pobreza extrema até o ano de 2015, cujo quarto objetivo tinha como meta à redução em 2/3 das taxas de mortalidade na infância em relação ao nível de 1990 (Ipea, 2014). O alcance desta meta pelo Brasil antes do prazo previsto deveu-se, em parte, a alterações em indicadores sociais, principalmente os que se relacionavam à renda e ao desenvolvimento de ações assistenciais de saúde (Marinho et al., 2020).

Com foco na quarta meta dos ODM, ainda anos 2000 foi lançado o Método Canguru, em busca da humanização da assistência ao recém-nascido, atuando como uma importante estratégia no enfrentamento da mortalidade infantil, especificamente no componente neonatal, mediante a incorporação de tecnologias simples e práticas humanizadas na unidade de terapia intensiva neonatal, para garantir a sobrevida de recém-nascidos pré-termo ou gravemente enfermos. Pesquisa financiada pelo Ministério da Saúde, em 2005, com dezesseis unidades de saúde que contemplavam ou não o Método Canguru, incluindo 985 recém-nascidos pesando entre 500 e $1749 \mathrm{~g}$, verificou desempenho superior das unidades cangurus, que apresentaram menores percentuais de reinternação (9,6\% versus $17,1 \%)$, melhora quanto à prática do aleitamento materno exclusivo na alta (69,2\% versus $23,8 \%$ ), bem como 3 meses após a alta (Sanches et al., 2015).

Diante da compreensão de que a proposta de humanização da atenção à saúde era tida como uma das estratégias para responder efetivamente às complexas necessidades de saúde individuais e coletiva, em 2001 foi instituído o Programa Nacional de Humanização da Atenção Hospitalar (PNHAH) e o Programa Nacional de Triagem Neonatal (PNTN) para requalificar os hospitais públicos e promover o rastreamento populacional por meio da identificação de distúrbios e doenças no recém-nascido, respectivamente (Brasil, 2016; Brasil, 2018). Em 2003 foi desenvolvida a Política Nacional de Humanização (PNH) como estratégia de suporte emocional para as parturientes, visando aumentar a afetividade entre elas e os profissionais de saúde, favorecendo um ambiente mais humanizado e um parto mais seguro (Brasil, 2018).

Cabe ressaltar que nesse período a saúde registrava uma situação antagonista, com avanços institucionais em programas específicos de um lado e a persistência de graves desigualdades sociais de outro. Dessa forma, surgem em 
2001 o Programa Nacional de Renda Mínima, o Bolsa Alimentação (PBA), e o Programa Fome Zero (PFZ) como complementação da renda familiar e política de segurança alimentar para a melhoria da alimentação de gestantes, nutrizes e crianças de seis meses a seis anos e onze meses de idade (Brasil, 2001; Santos, Pasquim, Santos, 2011).

Em 2002 foi instituído o programa "Auxílio-Gás", destinado a subsidiar o preço do gás liquefeito de petróleo às famílias de baixa renda (Brasil, 2002). Em 2003, o Programa Nacional de Acesso à Alimentação, Cartão Alimentação (PCA) (Brasil, 2003). Posteriormente, alguns programas sociais como o Bolsa Alimentação, Auxílio Gás, Bolsa Escola sofreram unificação, momento em que é criado o Programa Bolsa Família (PBF), como instrumento para prover recurso financeiro às famílias em situação de insegurança alimentar ou de extrema pobreza. O PBF é um dos maiores programas de transferência condicional de renda no mundo, tendo atingido uma alta cobertura na última década, alcançando todos os 5.565 municípios brasileiros (Silva Paes, 2019), além de favorecer a diminuição da pobreza e fomentar a maior utilização dos serviços de saúde ofertados às famílias pelo SUS (Ipea, 2014). Programas de transferência de renda ajudam a reduzir as mortes na infância ao contribuírem com um acesso mais adequado aos serviços de saúde e educação para as famílias, condicionando a sua permanência (Abrasco, 2018).

Entre 2003 e 2008 as políticas de redução da pobreza receberam destaque governamental, fato que contribuiu para a redução da desigualdade de renda no país (Teixira, Paim, 2005), diminuindo o risco de morte das crianças. Sucessivamente, no ano de 2004, com a perspectiva de ajudar os gestores estaduais e municipais no processo de reorganização da rede de assistência à infância nos seus vários níveis, foi instituída a Agenda de Compromissos para a Saúde Integral da Criança e Redução da Mortalidade Infantil (Brasil, 2004) e firmado o Pacto pela Redução da Mortalidade Materna e Neonatal, com a ampliação de um conjunto de ações estratégicas visando à melhoria do cuidado perinatal (Brasil, 2018). Nesse mesmo ano foi instituído o Comitê Nacional de Prevenção do Óbito Infantil e Fetal, a partir de um movimento caracterizado pela responsabilização da prevenção das mortes precoces e evitáveis (Brasil, 2004). Outra importante ação do Ministério da Saúde foi a instituição da Caderneta de Saúde da Criança, Passaporte da Cidadania, como ferramenta estratégica para o acompanhamento do crescimento e desenvolvimento e de orientações para a atenção integral das crianças até os seus 9 anos de vida (Brasil, 2018).

No início de 2006 foi consolidado o Pacto pela Saúde por meio da Portaria n 399 do Ministério da Saúde, representando um conjunto de reformas institucionais, com o objetivo de promover inovações nos processos e instrumentos de gestão. Para isso, as três esferas de gestão do SUS (Município, Estado e União) realizaram pactuação. O instrumento para formalização dos compromissos entre gestores foi o Termo de Compromisso de Gestão que definiu metas articuladas e integradas nos três componentes: Pacto pela Vida, Pacto em Defesa do SUS e Pacto de Gestão do SUS. O Pacto pela Vida tinha como uma de suas prioridades a redução da mortalidade materna, infantil neonatal, infantil por doença diarreica e por pneumonias (Brasil, 2006; Menicucci, Costa, Machado, 2018).

Foi nesse mesmo ano que surgiu a Política Nacional de Atenção Básica (PNAB), prioritária para expansão, qualificação e consolidação da Atenção Básica de Saúde (ABS) no país, passando a denominar o PSF de Estratégia de Saúde da Família (ESF). A PNAB explicita a Saúde da Família como sendo a estratégia prioritária para expansão, qualificação e consolidação da ABS no país, considerando a ABS como uma potente ferramenta capaz de gerar impacto na situação de saúde de indivíduos e coletividades e de ampliar a resolutividade de problemas, além de propiciar uma importante relação custo-efetividade, em função dos recursos necessários para sua implementação. Em 2011 e 2017 a PNAB foi revisada (Gonçalves Junior, Gava, Silva, 2017; Brasil, 2017c).

As principais modificações realizadas na PNAB em 2017 foram a ampliação das atribuições dos agentes comunitários de saúde; a possibilidade de financiamento de outros modelos de organização da ABS, além da ESF; a inclusão do gerente de ABS nas equipes e a construção da oferta nacional de serviços e ações essenciais e ampliadas da 
ABS4. Tais mudanças, em meio a uma conjuntura de fortalecimento da ideologia neoliberal, reafirmam o processo de desconstrução do SUS e a subtração de direitos conquistados46. Cabe destacar que uma análise sobre a mortalidade infantil e o PSF nas unidades da Federação brasileira, de 1998-2008, mostrou que 73\% dos estados brasileiros apresentaram correlação negativa entre o aumento na cobertura populacional e a redução da mortalidade infantil ( $\mathrm{p}<0,01)$ (Ceccon et al., 2014).

A partir de 2007 foi criado o Programa de Aceleração do Crescimento (PAC). Este teve como foco o estímulo ao crescimento da economia do país, por meio da destinação de recursos para áreas prioritárias de infraestrutura. Porém, apesar do grande investimento na área do saneamento básico, a concepção e a implementação do PAC apresentou uma série de contradições e fragilidades, especialmente para fazer frente aos desafios da universalização dos serviços no país (Cunha, Borja, 2018). Estudo sobre o impacto de ações assistenciais e mudanças socioeconômicas e sanitárias na mortalidade de crianças, após o estabelecimento das metas dos ODM, evidenciou grande influência das condições sanitárias na proposição de um modelo de regressão múltipla, sugerindo que este indicador contribui para o acesso e cuidado à saúde das crianças (Marinho et al., 2020).

Isto posto, os determinantes contextuais quando somados às ações de saúde são importantes influenciadores na determinação da mortalidade na infância. Sendo assim, em 2008 foi instituída a Rede Amamenta Brasil, uma estratégia nacional de promoção, proteção e apoio ao aleitamento materno, direcionada à ABS. Em seguida, passou a ser denominada Estratégia Amamenta e Alimenta Brasil, em 2013, após integração com a Estratégia Nacional de Promoção da Alimentação Complementar Saudável (Brasil, 2008b, Brasil, 2013).

Posteriormente, em 2009, foram desenvolvidos a Estratégia Brasileirinhas e Brasileirinhos Saudáveis: Primeiros Passos para o Desenvolvimento Nacional (Estratégia Brasileirinhos), que serviu para o fortalecimento e enfrentamento das iniquidades que interferem na saúde da mulher e da criança na primeira infância, e o Programa Compromisso Mais Nordeste e Mais Amazônia Legal pela Cidadania, visando diminuir as desigualdades regionais e reduzir em 5\% ao ano as taxas de mortalidade neonatal e infantil em 256 municípios prioritários (Brasil, 2018).

Seguindo essa tendência, em 2011 é lançada a Rede Cegonha tendo como uma de suas metas a redução da mortalidade materna e infantil com ênfase no componente neonatal (Brasil, 2011c) Nesse período, surgem algumas estratégias de qualificação e fixação dos profissionais da saúde em áreas mais vulneráveis e desprovidas de uma assistência qualificada. Assim, foi instituído o Programa de Valorização do Profissional da Atenção Básica (PROVAB), para estimular e valorizar o profissional de saúde das equipes multiprofissionais no âmbito da ABS e da ESF e levá-los para localidades com maior carência para este serviço (Brasil, 2011d).

Neste mesmo ano ressurge a preocupação com questões relacionadas à pobreza, ocasião em que é implantado o Plano Brasil Sem Miséria (BSM), com o objetivo ambicioso de superar a extrema pobreza até o final de 2014 (Ipea, 2020). No entanto, apesar do BSM ter logrado êxito em algumas de suas ações, não conseguiu alcançar a sua finalidade maior, com a extrema pobreza voltando a ter seus níveis elevados a partir de 2015.

No âmbito da saúde foi criado o Programa Nacional de Melhoria do Acesso e da Qualidade da Atenção Básica (PMAQ-AB), também em 2011, para incentivar os gestores e as equipes de saúde do SUS a melhorarem o padrão de acesso e da qualidade da assistência oferecida aos usuários nas ESF (Brasil, 2012). O PMAQ-AB oportunizou o acompanhamento e aprimoramento das atividades de coordenação do cuidado enfatizando que esse atributo interfere na qualidade da assistência à criança (Cruz et al., 2019). Na sequência, em 2012 foi lançada a ação Brasil Carinhoso, cujo propósito foi a prevenção da deficiência de vitamina A, da anemia e da ocorrência de mortes por diarreia e outras doenças infecciosas em crianças entre 6 meses e 5 anos de idade atendidas na ABS (Brasil, 2014). 
No ano seguinte, em 2013, dando continuidade à política de enfrentamento à falta de médicos na ABS, foi criado o Programa Mais Médicos (PMM), mediante a formação de recursos humanos na área médica para o SUS. De 2013 a 2015 a cobertura populacional da ABS no Brasil passou de 80,6\% a 88,8\%, o que representa um crescimento médio/anual quatro vezes maior que o crescimento médio dos cinco anos anteriores (Pinto et al., 2017).

Em seguida, em 2015, foi instituída a Política Nacional de Atenção Integral à Saúde da Criança (Pnaisc) visando promover e proteger a saúde da criança e a redução da morbimortalidade (Brasil, 2018). Nesse mesmo ano, a ONU lança os Objetivos de Desenvolvimento Sustentável (ODS), com uma agenda de ação até 2030, com destaque para a redução da mortalidade neonatal para pelo menos 12 por $1.000 \mathrm{NV}$ e a mortalidade de crianças menores de 5 anos para pelo menos 25 por $1.000 \mathrm{NV}$ no mundo até 2030 (OPAS, 2020).

Assim, pode-se perceber que durante todos esses anos a saúde da criança passou por um processo de grande evolução e aprimoramento, atraindo cada vez mais atenção quanto ao desenvolvimento de políticas públicas nacionais e internacionais, com foco na redução das TMI e TMInf.

\section{Considerações Finais}

Ao longo da história do Brasil o país apresentou uma série de estratégias, programas e políticas públicas de saúde e de âmbito social que, de forma direta ou indireta, contribuíram com a melhora das condições de saúde e sobrevida de muitas crianças brasileiras favorecendo a redução nas TMI e TMInf. Avanços foram obtidos e precisam ser comemorados, no entanto, ainda existe um longo caminho a ser percorrido em busca da redução das iniquidades socioeconômicas e regionais que influenciam diretamente a saúde das crianças e aumentam o risco de mortalidade desse grupo.

O recuo da cobertura vacinal, fato observado no país desde o início dos anos 2000 (Gaiva, 2021) com agravamento nos últimos anos, em face da fragilidade política e econômica, do movimento crescente anti-vacinas, com divulgação de informações falsas e com a falsa sensação de segurança em relação às doenças (Abrasco, 2018) aumenta o risco do retorno de epidemias de doenças anteriormente erradicadas.

Ademais, o cenário atual continua cheio de incertezas para a área da saúde, as medidas de austeridade fiscal adotadas desde 2015, em meio a uma crise política e econômica previamente estabelecida, acentuaram ainda mais o subfinanciamento do SUS, comprometendo muitas de suas ações de saúde. Além disso, a pandemia do COVID-19 tornou mais explícito a precariedade das condições de vida da maioria da população brasileira e a fragilidade da rede de proteção social, reforçando a necessidade da manutenção e da proposição de políticas de proteção à vida capazes de agir sobre os determinantes sociais da saúde que interferem na morbimortalidade na infância, de modo a garantir a sustentabilidade das ações para os próximos anos.

Assim, recomenda-se o desenvolvimento de novos estudos que promovam o acompanhamento da tendência das TMI e TMInf, bem como das políticas públicas que atuam na sua determinação. Destaca-se ainda a necessidade de investimentos para a manutenção e ampliação destas políticas públicas e do SUS para que possamos continuar avançando quanto à redução das mortalidades infantil e na infância.

\section{Referências}

Almeida, E. R., Sousa, A. N. A., Brandão, C. C., Carvalho, F. F. B., et al (2018). Política Nacional de Atenção Básica no Brasil: uma análise do processo de revisão (2015-2017). Revista Panamericana de Salud Pública. 42: e180.

Araújo, J. P., Araújo, J. P., Silva, R. M. M., et al (2014). História da saúde da criança: conquistas, políticas e perspectivas. Revista Brasileira de Enfermagem. 67 (6): 1000-1007. 
Abrasco - Associação Brasileira de Saúde Coletiva (2018). Especial Abrasco sobre o aumento da mortalidade infantil e materna no Brasil. Rio de Janeiro: Associação Brasileira de Saúde Coletiva. <https://www.abrasco.org.br/site/noticias/institucional/especial-abrasco-sobre-o-aumento-da-mortalidade-infantil-ematerna-no-brasil/36777/>

Brasil (1997). Ministério da Saúde. Metas da Cúpula Mundial em Favor da Infância: avaliação de meia década, 1990-1995. Ministério da Saúde.

Brasil (2001). Medida Provisória No 2.206, de 10 de agosto de 2001. Cria o Programa Nacional de Renda Mínima vinculado à Saúde: Bolsa-Alimentação. Diário Oficial [da] República Federativa do Brasil, Brasília, DF, 13 ago. 2001.

Brasil (2002). Ministério da Saúde. A Política de Saúde no Brasil nos anos 90: avanços e limites. Ministério da Saúde.

Brasil (2003). Medida Provisória No. 108 de 27 de fevereiro de 2003. Cria o Programa Nacional de Acesso à Alimentação - "Cartão Alimentação". Diário Oficial [da] República Federativa do Brasil, Brasília, DF, 28 fev. 2003.

Brasil (2004). Ministério da Saúde. Secretaria de Atenção à Saúde. Departamento de Ações Programáticas Estratégicas. Agenda de compromissos para a saúde integral da criança e redução da mortalidade infantil. Brasília, DF: Ministério da Saúde.

Brasil (2005). Estatuto da Criança e do Adolescente: disposições constitucionais pertinentes: lei no 8.069, de 13 de julho de 1990. Brasília, DF: Ministério da Saúde.

Brasil (2006). Portaria $n^{\circ}$ 399, de 22 de fevereiro de 2006. Divulga o Pacto pela Saúde 2006 - Consolidação do SUS e aprova as Diretrizes Operacionais do Referido Pacto. Diário Oficial de União 2006, Brasília, DF, 22 fev. 2006.

Brasil (2008a). Ministério da Saúde. Sistema Único de Saúde. Painel de Indicadores do SUS nº 4. Temático Saúde da Família v II. Brasília: MS.

Brasil (2008b). Portaria $n^{\circ}$ 2.799, de 18 de novembro de 2008. Institui, no âmbito do Sistema Único de Saúde - SUS -, a Rede Amamenta Brasil. Diário Oficial de União. Brasília, 18 nov. 2008.

Brasil (2011a). Ministério da Saúde. Secretaria de Atenção à Saúde. Área Técnica de Saúde da Criança e Aleitamento Materno. Gestões e gestores de políticas públicas de atenção à saúde da criança: 70 anos de história. Brasília, DF: Ministério da Saúde.

Brasil (2011b). Ministério da Saúde. Secretaria-Executiva. Subsecretaria de Assuntos Administrativos. SUS: a saúde do Brasil. Brasília, DF: Ministério da Saúde.

Brasil (2011c). Ministério da Saúde. Secretaria de Atenção à Saúde. Departamento de Ações Programáticas Estratégicas. Atenção humanizada ao recémnascido de baixo peso: Método Canguru. Brasília, DF: Ministério da Saúde.

Brasil (2011d). Portaria interministerial nº 2.087, de $1^{o}$ de setembro de 2011. Institui o Programa de Valorização do Profissional da Atenção Básica. Diário Oficial da União.

Brasil (2012). Ministério da Saúde. Secretaria de Atenção à Saúde. Departamento de Atenção Básica. Programa Nacional de Melhoria do Acesso e da Qualidade da Atenção Básica (PMAQ): manual instrutivo. < http://189.28.128.100/dab/docs/publicacoes/geral/manual_instrutivo_pmaq_site.pdf>

Brasil (2013). Portaria $n^{o}$ 1.920, de 5 de setembro de 2013. Institui a Estratégia Nacional para Promoção do Aleitamento Materno e Alimentação Complementar Saudável no Sistema Único de Saúde (SUS) -Estratégia Amamenta e Alimenta Brasil. Diário Oficial de União. Brasília, 5 set. 2013.

Brasil (2014). Ministério da Saúde. Secretaria de Atenção à Saúde. Departamento de Ações Programáticas Estratégicas. Atenção à saúde do recém-nascido: guia para os profissionais de saúde. 〈http://bvsms.saude.gov.br/bvs/publicacoes/atencao_saude_recem_nascido_v1.pdf>

Brasil (2016). Ministério da Saúde. Secretaria de Atenção à Saúde. Departamento de Atenção Especializada e Temática. Triagem neonatal biológica: manual técnico. Brasília, DF: Ministério da Saúde.

Brasil (2017a). Ministério da Saúde. Secretaria de Atenção à Saúde. Departamento de Ações Programáticas Estratégicas. Bases para a discussão da Política Nacional de Promoção, Proteção e Apoio ao Aleitamento Materno. Brasília, DF: Ministério da Saúde.

Brasil (2017b). Ministério da Saúde. Manual de quadros de procedimentos: Aidpi Criança: 2 meses a 5 anos. Ministério da Saúde

Brasil (2017c). Ministério da Saúde. Política Nacional de Atenção Básica. Ministério da Saúde.

Brasil (2018). Ministério da Saúde. Secretaria de Atenção à Saúde. Departamento de Ações Programáticas Estratégicas. Política Nacional de Atenção Integral à Saúde da Criança: orientações para implementação. Ministério da Saúde.

Brasil (2020a). Ministério da Saúde. Secretaria de Vigilância em Saúde. Departamento de Informação e Análise Epidemiológica. Indicadores de Mortalidade que utilizam a metodologia de Busca Ativa. Ministério da Saúde. 
Brasil (2020b). Ministério da Saúde. Secretaria de Atenção Primária à Saúde (SAPS). Estratégia de Saúde da Família (ESF). Agente Comunitário de Saúde. < https://aps.saude.gov.br/ape/esf/esf/composicao >

Cacau, M. P., Rodrigues, L. S., Rêgo, A. S., et al (2015). Mortalidade em crianças menores de 10 anos no Maranhão. Rev Pesq Saúde, 16:166-169.

Caldas, A.D. R., Santos, R. V., Borges, G. M., et al (2017). Mortalidade infantil segundo cor ou raça com base no Censo Demográfico de 2010 e nos sistemas nacionais de informação em saúde no Brasil. Cad. Saúde Pública, 33: e00046516.

Ceccon, R. F., Bueno, A. L. M., Hesler, L. Z., Kirsten, K. S., et al (2014). Mortalidade infantil e Saúde da Família nas unidades da Federação brasileira, 19982008. Cad. saúde colet., 22:177-183.

Cordeiro, A. M., Oliveira, G. M., Rentería, J. M., \& Guimarães, C. A. (2007). Revisão sistemática: uma revisão narrativa. Revista do Colégio Brasileiro de Cirurgiões, 34 (6): 428-431.

Costa, R., Padilha, M. I., Monticelli, M., et al (2010). Políticas Públicas de Saúde ao recém-nascido no Brasil: reflexos para a assistência neonatal. Hist Enferm Rev Eletronica, 1:55-68.

Cruz, M. J. B., Santos, A. F., Araújo, L. H. L., et al (2019). A coordenação do cuidado na qualidade da assistência à saúde da mulher e da criança no PMAQ. Cad. Saúde Pública, 35(11).

Cunha, M. A., \& Borja, P. C. (2018). O programa de aceleração do crescimento no estado da Bahia e os desafios da universalização do saneamento básico. Revista Brasileira de Gestão Urbana, 10(1):173-185.

Escorel, S. (1999). O Programa de Interiorização de Ações de Saúde e Saneamento (Piass). In: Escorel, S. Reviravolta na saúde: origem e articulação do movimento sanitário [online]. Editora Fiocruz. p.161-78

Gaiva, M. A. M. (2021). Imunização no contexto da pandemia de COVID-19. Revista Nursing. 24 (272): 5074.

Gonçalves Junior, O., Gava, G. B., \& Silva, M. S. (2017). Programa Mais Médicos, aperfeiçoando o SUS e democratizando a saúde: um balanço analítico do programa. Saude soc., 26 (4): 872-887.

Guimarães, L. M. B., \& Silva, S. J. (2020). I Plano Nacional de Segurança Alimentar e Nutricional e o Bolsa Família em perspectiva intersetorial. Serv. Soc. Soc., 137:74-94.

Hochman, G. (2011). Vacinação, varíola e uma cultura da imunização no Brasil. Ciênc. saúde coletiva, 16 (2): $375-386$.

Ipea - Instituto de Pesquisa Econômica Aplicada (2014). Objetivos de Desenvolvimento do Milênio: relatório nacional de acompanhamento.

Ipea - Instituto de Pesquisa Econômica Aplicada (2020). Inovação no setor público. Plano Brasil sem Miséria. <https://www.ipea.gov.br/labgov /inovacoes/case s/66-estrategia-de-monitoramento-analitico-do-plano-brasil-sem-miseria-e-programas-do-mds-2>

Lima, A. A., \& Pinto, E. S. (2017). O contexto histórico da implantação do Programa Nacional de Imunização (PNI) e sua importância para o Sistema Único de Saúde (SUS). Scire Salutis., 7(1): 53-62.

Magalhães, M. L., Cannon, L. R. C., Coimbra, T. S., \& Padilla, H. (2018?). Desafios da mortalidade infantil e na infância. < https://apsredes.org/pdf/sus-30anos/03.pdf>

Marinho, C. S. R., Flor, T. B. M., Pinheiro, J. M. F., et al (2020). Objetivos de Desenvolvimento do Milênio: impacto de ações assistenciais e mudanças socioeconômicas e sanitárias na mortalidade de crianças. Cad. Saúde Pública., 36(10): e00191219.

Menicucci, T. M. G., Costa, L. A., \& Machado, J. Á. (2018). Pacto pela saúde: aproximações e colisões na arena federativa. Ciênc. saúde coletiva, 23:29-40.

Morosini, M. V. G. C., Fonseca, A. F., Lima, L. D. Política Nacional de Atenção Básica 2017: retrocessos e riscos para o Sistema Único de Saúde. Saúde em Debate, 2018; 42 (116): 11-24.

Nascimento, D. R. (2011). As campanhas de vacinação contra a poliomielite no Brasil (1960-1990). Ciênc. saúde coletiva, 16: 501-511.

Organização Pan-Americana de Saúde (2021). Brasil. Objetivos de Desenvolvimento Sustentável. https://www.paho.org/bra/index.php?option=com_con tent\&view=article\&id=5849: objetivos-de-desenvolvimento-sustentavel\&Itemid=875

Pinto, H. A, Oliveira, F. P, Santana, J. S. S, Santos, F. O. S., Araujo, S. Q., Figueiredo, A. M., \& Araújo, G. D. (2017). Programa Mais Médicos: avaliando a implantação do Eixo Provimento de 2013 a 2015. Interface (Botucatu), 21(Suppl 1):1087-1101.

Rasella D, Basu S, Hone T, et al (2018). Child morbidity and mortality associated with alternative policy responses to the economic crisis in Brazil: A nationwide microsimulation study. PLoS Med., 15: e1002570. 
Research, Society and Development, v. 10, n. 11, e474101119584, 2021

(CC BY 4.0) | ISSN 2525-3409 | DOI: http://dx.doi.org/10.33448/rsd-v10i11.19584

Sanches, M. T. C. et al (2015). Método Canguru: 15 anos da política pública. São Paulo: Instituto de Saúde. <https://portaldeboaspraticas.iff.fiocruz.br/wpcontent/uploads/2017/10/canguru_capa_miolo.pdf>

Santos, A. M. A., \& Jacinto, P. A. (2008a). O Impacto do Programa Saúde da Família Sobre a Saúde das Crianças da Área Rural do Brasil. Rev. Econ. Sociol. Rural., 55: 227-246.

Santos, I. S., Matijasevich, A., Gorgot, L. R. M. R., et al (2014). Óbitos infantis evitáveis nas coortes de nascimentos de Pelotas, Rio Grande do Sul, Brasil, de 1993 e 2004. Cad. Saúde Pública, 30:2331-2343.

Santos, L. M. P., Pasquim, E. M., \& Santos, S. M. C. (2011). Programas de transferência de renda no Brasil: um estudo multidimensional da implementação do Bolsa Escola, Bolsa Alimentação e Cartão Alimentação. Ciênc. saúde coletiva, 16:1821-1834.

Silva, E. S. A., \& Paes, N. A. (2019). Programa Bolsa Família e a redução da mortalidade infantil nos municípios do Semiárido brasileiro. Ciênc. saúde coletiva, 24:623-630.

Silva, J. G., Grossi, M. E. D., França, C. G. (2010). Fome Zero: A experiência brasileira; MDA. <http://nead.mda.gov.br/download.php?file=public acoes/especial/fome_zero_a_experiencia_brasileira.pdf>

Silva Junior, J. B. (2013). 40 anos do Programa Nacional de Imunizações: uma conquista da Saúde Pública brasileira. Epidemiol. Serv. Saúde, 22(1): 7-8.

Teixeira, C. F., \& Paim, J. S. (2005). A política de saúde no Governo Lula e a dialética do menos pior. Saúde Debate, 29:268-83.

Viana, A. L. D., \& Poz, M. R. D. (2005). A Reforma do Sistema de Saúde no Brasil. PHYSIS: Rev. Saúde Coletiva, 15(Supl):225- 264. 